com loose from the walls of the abdomen from a point midway down the pelvis to the diaphragm, and from the rectus muscle anteriorly to the vertebra posteriorly. The left kidney had been displaced and was apparently floating, and the entire descending colon, together with the balance of the abdominal viscera which should occupy the left side, were pushed over to nearly the median line, and were enveloped in a sack of loose peritoneum. There was not the slightest evidence of peritonitis, the bowels being flat, and apparently showing no signs of irritation.

There being considerable hemorrhage in the form of capillary oozing, I carried a second incision at right angles with the first, for the purpose of drainage, cutting through the abdominal muscles, to the outer border of the lumbar muscles. After thoroughly cleansing this cavity, I drenched the parts with hydrogen peroxid, after which it was packed with a large quantity of gauze soaked in warm bichlorid evaporating solution. The patient, although weak and exhausted before the operation, rallied from it very nicely.

The day following the operation he seemed to be semiconscious, which seemed to be the result of exhaustion. as there was no evidence of inflammatory trouble, and little or no rise of temperature. There was no effort at repair, and whilst the wound remained perfectly clean, there was a peculiar ammoniacal odor connected with it, although there was no evidence of escape of urine into the wound from either the kidney, ureter or bladder; this condition, to my mind, indicated molecular dissolution. There was incontinence of urine, and whilst the patient took considerable nourishment, which apparently seemed to digest, yet he gradually sank and died on December 11. just five days after the operation and twentyseven days after the accident.

\section{GENERAL COMMENTS.}

We have had here a series of injuries in which there were grave abdominal injuries without corresponding external evidences of traumatism, yet the result in each case was fatal. In four of the cases reported, we have had rupture of some part of the alimentary canal, followed by death. What are the lessons we are to learn from these grave accidents? Could the lives of these patients have been saved through operative interference?

In the first case, in which the patient was struck by a paling: I firmly believe that if operative interference had been resorted to immediately after the accident, that the patient would have survived the injury. In the case of rupture of the stomach, there was such general infection of the entire abdominal cavity, as shown by the postmortem, such rapid collapse, that I question whether it would have been possible to have saved the life of this patient unless the surgeon had been actually on the ground at the time, and made immediate operation. It must be remembered, as in this case, that these accidents frequently occur miles from surgical aid, and by the time the surgeon reaches the patient the time for active interference has past.

This applies to the third case, in which the patient ran violently against the end of a rail, producing rupture of the intestine, but as the patient was past all hope of recovery when seen by the writer, and no postmortem was permitted, it is only conjecture as to the real injury produced. At all events, operative interference would have failed in this case by the time it was possible for a surgeon to reach him.

In the Bohemian miner, the question of immediate operative interference might well be considered. This man was in the hospital in less than three hours from the time the accident occurred. The question of operative intervention came up, but inasmuch as the patient never rallied from shock, I do not consider it good surgery to undertake an abdominal operation without any knowlcdge of where the injury might be located, with a pulseless patient, suffering from extreme shock, from which he never rallied. Had I seen the slightest sign of rallying, I would have operated on him at once.

This, of course, brings up the mooted question of operating in shock, and although I have for many years advocated major amputations under shock, and have made many such amputations under such circumstances where the patient has rallied after the removal of a mangled limb, which I doubt ever would have rallied without its removal, I was skeptical in this case. In abdominal operations where there is severe shock, it seems to me that there is a different condition of affairs to contend with, and it is a question in my mind whether operative interference for grave abdominal injuries, with the patient suffering from the severest form of shock, is advisable, or would result in benefit to the patient except in hemorrhage.

The last case is a peculiar one, and brings up the question of operations for hematoma. It is my custom not to operate for hematoma as long as there is reasonable hope that they are being absorbed. I see many accidents every year in which there are severe hematoma located between the muscles, which are rapidly absorbed, and the patient gets well with little or no difficulty. Occasionally we have hematoma that do not absorb, which I consider proper cases for operative interference, and would treat such cases the same as I would a pocket of pus: evacuate and drain thoroughly. We admit, in these days of antiseptic and aseptic surgery. that incision into muscular tissue with evacuation of collections of blood, ought not to be followed by infection, but notwithstanding all the precautions the surgeon may resort to, these patients are liable to become infected. I seldom make compound of simple or open of closed wounds when it can be avoided, and particularly so where nature is inclined to take care of the extravasation by the natural channels of absorption. This is particularly true when we have different nationalities to deal with who do not readily understand or thoroughly comprehend the English language and are liable to unintentionally disregard the surgeon's instructions.

\section{CONCLUSION.}

From reading the literature of this class of cases, together with my own experience, I am led to the conclusion that it is the surgeon's duty to make an exploratory incision in all cases where there is grave doubt as to the real nature of the injury, and particularly so when the constitutional symptoms point to a condition more serious than is indicated by either the subjective or objective symptoms, provided the physical condition of the patient is such as to warrant an operative procedure.

\section{Glinical Report.}

\section{UNPRECEDENTED CASE OF CONSTIPATION. D. GEIB, M.D.. AND J. D. JONES, M.D. \\ GROTON, S. D.}

This case, reported to the South Dakota State Medical Society, June, 1900, and to the Aberdeen District Medical Society, June, 1901, by Dr. D. Geib, was further described in a communication to the Aberdeen District Medical Society, September, 1901, by Dr. J. D. Jones and concluded by report 
of the case to time of death, January 8, 1902, with autopsy, by Drs. D. Geib and J. D. Jones.

The patient, Mr. K., having enjoyed good health previous to the age of 11 , began at that time to be constipated and was treated for three months by Dr. Nicholas Senn, then of Dodge County, Wisconsin, no bowel movement being secured. The only result of cathartics and laxatives was severe pain. The constipation continued until death. It was a common occur rence for him to go three weeks or as many months without a movement of the bowels. At the age of 20 he did not have a movement for three or four months at a time. $\mathrm{He}$ con sulted a homeopathic physician, who prescribed for him two drops of croton oil, to be doubled in two hours, tripled in four hours, and quadrupled in six hours. This produced no result but after a period of several weeks, his bowels moved again.

For seven years following, his bowels were fairly regular. At the age of 29 he contracted a severe cold and five months and three days passed without an evacuation. After a few months of regularity, his bowels did not move for six months and fourteen days. At this time he consulted Dr. Stamm of St. Paul, without immediate benefit, but during the next six years his bowels were regular. In February, 1900, the consti pation returned. The patient had no movement from June 18, 1900 , to June 21,1901 .

During these periods of costiveness he could eat full meals and do a good day's work. His respiration was always nor mal: the urine was normal when he was free from pain, but

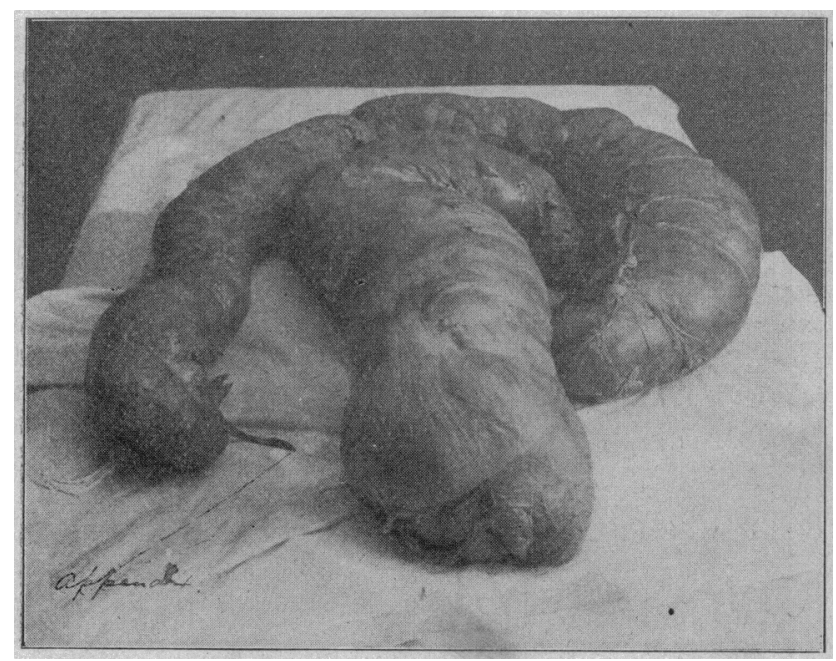

The colon of Mr. K

highly colored when in pain. The evacuation of his bowels made him very weak and he was greatly troubled with gas so that he had to lie on his right side to relieve himself and was partially disabled for work.

His abdomen was greatly distended so that the liver and the stomach crowded up the diaphragm and the floating ribs were visibly pushed out. There was tenderness in the sig. moid flexure, but over the remainder of the abdomen he could bear heavy pressure.

He was not troubled with gas when regular. The administration of valerian, sumbul and asafetida by Dr. Geib gave him considerable relief.

On June 19, 1901, Dr. Jones was called in with Dr. Geib to relieve the patient from his painful condition. The abdomen was greatly distended. The colon, on palpation, seemed to be as large as a six-inch stovepipe. From the head of the sigmoid flexure to the rectum, the bowel seemed to be perfectly straight and hard. On digital examination the rectum was found filled with a mass of fecal matter so hard that no impression could be made on it. The anus was dilated and the mass removed from the rectum with a bone curette and hot water. Further operation was postponed because of the pain suffered by the patien 1 , and olive oil enemas were ordered.

June 21 , on further attempt to evacuate the bowels,. it was found that the olive. oil had softened the hard mass so that about two pounds of the feces could be removed before the patient complained of pain; his weakened condition prevented further operation. The circumference of the patient at this time at the ensiform cartilage was 39 in., at the umbilicus, 38.5 in., and at the crest of the ileum, $39 \mathrm{in}$.

On arrival at the house on the morning of June 22 , the report was received that he had passed an ordinary pailful of feces since the day previous. There was much rejoicing in the family. The patient was very weak and sore, so no further operation was attempted at this time, but the olive oil enemas were ordered continued.

The patient, when next seen, on June 25, was feeling comfortable; the gas had ceased to trouble him and he had passed about three quarts of feces that morning. His measurements at this date were, at the ensiform cartilage 34 in., umbilicus 33 in., and at the crest of ileum 30 in.

The enemas were ordered continued. Mr. K. estimated that he had passed about eight gallons of feces since the beginning of the treatment. On June 29 , he was cheerful and pleased at his progress. The measurements at the ensiform cartilage were 34 in., umbilicus 30 in., crest of ileum 29 in. From that date he received massage treatment given by Dr. Geib, twice a week for three weeks, and improved in sirength so that he was able to ride to town and walk about. One July 8, after his massage, he suffered considerable pain for about three days.

His bowels moved frequently. The contents were described as resembling soft soap and during this period of discomfort he passed a hard mass about the size of a duck's egg, containing grape seeds. He had not eaten grapes since the fall before. After this he had little trouble and gained in strength and weight. The only treatment then given was massage, iron and stryehnia.

The history of the case subsequent to the above report to the Aberdeen District Medical Socicty is as follows: Since the treatment in June, 1901, the bowels moved regularly; occasionally he was obliged to take an enema, but he was well nourished and weighed more than he had for years. He was very sensitive to cold this winter, chilling on the least exposure.

On the second day before his death, which occurred Jan. 8 , 1902, he rode to town, a distance of eight miles. He retired at $11 \mathrm{p}$. m. apparently as well as usual, and awoke about 5 a. m. with pain. He arose about 8 a. m., built the fires and did part of his morning's work. The pain, however, became very intense and he went to the house and suffered more or less all day. There was a great desire to evacuate the bowels, but it was impossible. At 2 o'clock the following morning, he died while sitting on the stool. No medical aid was called.

The autopsy showed the abdomen greatly distended with gas and fecal matter. On making an incision along the linea alba, the tension was sufficient to tear the flesh apart; the omentum was very thin and the colon was brought at once into view. The position of the colon was as shown in the photograph, save that the extra loop overlaid the normal colon. The splenic thexure, transverse colon and descending portion of the extra loop were very much thickened, containing much more muscu lar fiber than nornial. The parts most distended were the splenic flexure, the transverse and the descending portion of the extra loop, but the whole colon was much larger than normal.

The most distended portion measured $191 / 4$ inches in circumference. The rectum contained a hard mass of feces shaped like a goose egg, measuring about 4 inches in the shoriest diameter and 6 in its longest. This was pressed tightly against the sphincter and acted as a valve. The remaining portion of the colon contained soft feces; the total contents were an ordinary bucketful. The stomach and small intestines were enpty. The diaphragm was crowded up to the level of the fourth rib on the right side; the heart and lungs were both displaced.

Iodoform Odor.-Oil of turpentine is suggested for the re moval of the odor of iodoform. It is claimed that it at once removes the objectionable smell from the hands or implements. -Med. Fortnightly. 